\title{
Effet des variations d'énergie lumineuse associées à l'agitation verticale sur le bilan photosynthétique de cultures intensives d'algues en bassin Effect of light energy variations and vertical mixing on photosynthetic budget for intensive outdoor algal cultures
}

\section{Bosca et A. Dauta}

Volume 4, numéro 3, 1991

URI : https://id.erudit.org/iderudit/705106ar

DOI : https://doi.org/10.7202/705106ar

\section{Aller au sommaire du numéro}

\section{Éditeur(s)}

Université du Québec - INRS-Eau, Terre et Environnement (INRS-ETE)

\section{ISSN}

0992-7158 (imprimé)

1718-8598 (numérique)

\section{Découvrir la revue}

\section{Citer cet article}

Bosca, C. \& Dauta, A. (1991). Effet des variations d'énergie lumineuse associées à l'agitation verticale sur le bilan photosynthétique de cultures intensives d'algues en bassin. Revue des sciences de l'eau / Journal of Water Science, 4(3), 381-392. https://doi.org/10.7202/705106ar

\section{Résumé de l'article}

Une série d'expériences a été réalisée pour évaluer les effets de l'agitation sur le bilan photosynthétique de cultures d'algues en masse en bassins extérieurs. La méthode utilisée compare la production d'échantillons marqués au $\mathrm{C}^{14}$ disposés (1) selon un profil vertical classique, (2) sur un système rotatif faisant transiter un flacon entre la surface et le tond du bassin. Des mesures ont été effectuées périodiquement en milieu de journée sur un cycle annuel, et ont été complétées par 8 incubations réalisées entre le lever et le coucher du soleil pour un jour donné. Dans toutes les expériences réalisées durant la période estivale, la photosynthèse est supérieure dans le flacon mobile. Les effets des variations lumineuses (par le biais des mouvements de l'eau) sur te bilan photosynthétique de la colonne d'eau sont particulièrement notables au lever et au coucher du soleil; ce bilan peut être jusqu'à 2.5 fois plus important pour une colonne d'eau en mouvement. Le calcul d'un bilan journalier par extrapolation des profils de photosynthèse instantanés permet d'estimer le gain de production obtenu par agitation du milieu. Par contre pendant la période hivernale, la photosynthèse est supérieure dans le flacon immobile. Ces résultats ont des implications évidentes sur le monitoring de cultures d'algues en masse en bassins extérieurs. 


\title{
Effet des variations d'énergie lumineuse associées à l'agitation verticale sur le bilan photosynthétique de cultures intensives d'algues en bassin*
}

\author{
Effect of light energy variations \\ and vertical mixing \\ on photosynthetic budget for intensive outdoor algal cultures
}

\section{BOSCA1 , A. DAUTA2}

Recu le 26 juiliet 1990 , accepté pour publication le 25 tévrier $1991^{\text {* * }}$.

RÉSUMÉ

Une série d'expériences a été réalisée pour évaluer les effets de l'agitation sur fe bilan photosynthétique de cultures d'algues en masse en bassins extérieurs. La méthode utilisée compare la production d'échantillons marqués au C14 disposés (1) selon un profil vertical classique, (2) sur un système rotatif faisant transiter un flacon entre la surface et le fond du bassin. Des mesures ont été effectuées périodiquement en milieu de journée sur un cycle annuel, et ont été complétées par 8 incubations réalisées entre le lever et le coucher du soleil pour un jour donné. Dans toutes les expériences réalisées durant la période estivale, la photosynthèse est supérieure dans le flacon mobile. Les effets des variations lumineuses (par le biais des mouvements de l'eau) sur le bilan photosynthétique de la colonne d'eau sont particulièrement notables au lever et au coucher du soleil ; ce bilan peut étre jusqu'à 2,5 fois plus important pour une colonne d'eau en mouvement. Le calcul d'un bilan lournaller par extrapolation des profils de photosynthèse instantanés permet d'estimer le gain de production obtenu par agitation du milieu. Par contre pendant la période hivernale, la photosynthèse est supérieure dans le fiacon Immobile. Ces résultats ont des implications évidentes sur le monitoring de cultures d'algues en masse en bassins extérieurs.

Mots clés : Production primaire, agitation, cultures d'algues.

1. Centre aquapure, 12160 Baraqueville.

2. Laboratoire d'tydrobiologie, U.P.S., 31062 Toulouse.

* Communication présentée au 34* Congrès de l'Association Française de Limnologie, Metz-Nancy, 28-30 mai 1990.

* Les commentaires seront reçus jusqu'au 30 mars 1992. 
Experiments were carrled out to define the effect of a particular mixing system (circular round a horizontal axis) upon the primary productivity of high-rate alga cultures. We know that mixing prevent algal cell sedimentation, gaseous and nutritional gradients, and variations in algae light reception. So, we wanted to quantify the effect of mixing on algal productivity with regard to flashing light (positive effect in comparison with continuous light).

We used water from waste stabilization ponds (secondary pond output), with the native algae population dominated by Euglena and Chlorella (in Aveyron, south of France). The method used compares algal production of a water column with "C-labelled samples i) in ten flasks placed along a classical vertical profile, ii) in a rotating system with a flask moving between the top and the bottom of the pond $(0.5 \mathrm{~m})$. The production is here considered as the mean of a mixed vertical profile. At the same time, physico-chemical and biological parameters were recorded (high load for $\mathrm{N}$ and $\mathrm{P}$ ).

Experiments were made periodically in the mid-afternoon over one year, and were completed by 8 incubations made between sunrise and sunset for on day (in August 1989). The aim was to evaluate the mixing effect with various environmental conditions. Physico-chemical parameters vary greatly over a day, and higher production values are measured in the mid-afternoon. Production observed for the stationary profile fitted the Steele model, and showed active photosynthetic from sunrise until sunset, with inhibition at high light intensities; maximal photosynthesis rates were measured between 4.00 and 5.00 PM.

Results are compared to moving flask values : in all the summer experiments photosynthesis was higher in the moving flask. Light variation effects (through water movements) on the water column photosynthetic budget are particularly notable at sunrise and sunset hours : this budget may be over 2.5 times that of homogeneous nonturbulent water. During winter however, photosynthesis is higher for the stationary profile. We compared production recorded with the two systems, and calculated the mathematical relationship between the two systems over one day (exponential) and over the year (linear relationship).

By extrapolation of the instant photosynthesis profile to a 24-hour period, we can evaluate the production gain with culture mixing. We calculated the net production for various days over the year; around the end of April, the mixed system became more productive than the stationary one.

Considering our results in environmental conditions, it is obvious that they are not representative of all mixing systems, particularly the techniques in which algae cell position shift continualiy with respect to the photic zone : the positive effect of mixing in our ponds may be linked to the flashing light effect. In the literature, we can see that photosynthetic activity increases with flash time duration. Our production figures for various hours along the day (or various flash durations) are in agreement with these results.

During low photosynthetic activity periods, the flashing light effect can not act since light is avallable at all depths of pond. The most important factor acting, in our experiments, seems to be light, since the two systems are in similar conditions.

These results have clear implications for monitoring intensive outdoor algal cultures.

Key-words : Primary production, mixing, algal culture. 


\section{INTRODUCTION}

L'effet de l'agitation sur la culture en masse des micro-algues est encore peu étudié tant au niveau des aspects fondamentaux que techniques du problème, même si très tôt des études ont montré son action positive sur la productivité algale (WINOKUR, 1948). En effet, l'agitation évite :

- la sédimentation des cellules algales (OSWALD, 1988),

- la présence de gradients gazeux et nutritionnels dus à l'activité métabolique,

- une disparité de l'énergie lumineuse reçue par les individus.

Des expériences faites en laboratoire, afin d'étudier les relations entre l'activité photosynthétique et l'agitation, démontrent l'effet positif de flashs lumineux en comparaison avec une lumière continue (MARKL, 1980, KOK et al. 1953, LITTLE, 1964, MYERS et GRAHAM, 1971, MYERS, 1980, TAMIYA et al., 1953). En ce qui concerne les cultures en masse en bassins extérieurs, RICHMOND et VONSHAK (1978) ont observé des variations de la productivité selon l'intensité d'agitation appliquée. Notre terrain d'étude, avec plusieurs bassins à grande échelle, nous a permis de quantifier l'effet d'un type d'agitation (circulaire dans le plan vertical) sur l'activité photosynthétique, en comparaison avec une culture non agitée.

\section{MATÉRIEL ET MÉTHODES}

\section{Terrain d'étude}

Les expérimentations ont eu lieu au Centre de Recherche AQUAPURE (Aveyron). Nous avons utilisé des bassins de $25 \mathrm{~m}^{3}$ (50 $\mathrm{cm}$ de profondeur) construits pour la culture en masse de microalgues. Ces bassins reçoivent l'effluent d'une lagune d'épuration, et les bassins de cultures sont équipés de roues à aubes (en mouvement 24 heures sur 24).

Les espèces présentes dans ces bassins sont des populations naturelles dominées par des Euglènes et des Chlorelles.

\section{Paramètres physico-chimiques}

La température, le $\mathrm{pH}$, l'oxygène et l'intensité lumineuse incidente sont mesurés ponctuellement ou enregistrés en continu. Ces paramètres sont homogènes autour du bassin et sur sa profondeur. Le coefficient d'extinction de la lumière dans l'eau est déterminé par comparaison de l'énergie lumineuse reçue au niveau de deux cellules photoélectriques, calibrées avec un quantamètre, et immergées à $6 \mathrm{~cm}$ et $20 \mathrm{~cm}$ de profondeur dans les cultures. 
Les charges moyennes en nutriments du milieu sont les suivantes :

- Ptotal $=12 \mathrm{mg} . \mathrm{I}^{-1}$

$-\mathrm{NO}_{2}$ et $\mathrm{NO}_{3}=0$

$-\mathrm{NH}_{4}=$ varie entre 100 et $200 \mathrm{mg} . \mathrm{H}^{-1}$ suivant les saisons.

\section{Paramètres biologiques}

Pour la détermination de la chlorophylle $a$, nous avons employé une méthode spectrophotométrique (MARKER et al., 1980).

L'activité photosynthétique est mesurée dans des flacons en verre de $20 \mathrm{ml}$, après ajout de $55 \mathrm{Kbq}$ de $\mathrm{NaH}^{14} \mathrm{CO}_{3}$ (CEA, Saclay). Après une heure d'incubation, une fraction aliquote $(4 \mathrm{ml}$ ) est passée (et rinçée avec de l'eau bi-permutée) sur des filtres HA Millipore de $0,45 \mu \mathrm{m}$. Ces filtres sont ensuite placés dans des flacons remplis de liquide à scintillation (Ready solv HP/b Beckman), et la radioactivité. corrigée pour le quenching, est enregistrée par un compteur Beckman LS $5000 \mathrm{CE}$. Les valeurs sont calculées en soustrayant la radioactivité du flacon incubé à l'obscurité :

$$
P b=\frac{A(t)-A D^{* 12} C^{*} 1.06^{*} 1 / t}{A !}
$$

$\mathrm{Pb}$ : production primaire (mg C.t.. $\left.\mathrm{h}^{-1}\right)$

$A(t)$ : activité au temps $t$ (DPM)

$A D$ : activité du flacon noir (DPM)

$\mathrm{Al}$ : activité introduite (DPM)

${ }^{12} \mathrm{C}$ : carbone minéral (mg..$^{-1}$ )

1.06 : constante de discrimination

$t$ : durée d'incubation $(H)$

La relation entre l'intensité lumineuse ( $($ ) et la photosynthèse $(P)$ est définie par les deux paramètres Pmax (capacité photosynthétique maximale en lumière saturante) et lopt (Intensité lumineuse de saturation) :

$$
P=P_{m a x}^{*} 1 / \text { lopt }^{*} I^{*} \exp (1-] 1 / \text { lopt }^{*} \mid
$$

\section{Principe expérimental et appareillage}

La production brute instantanée d'une colonne d'eau est estimée selon deux techniques (fig. 1). A partir d'un échantillon d'eau prélevé juste sous la surface en milieu de bassin, deux dispositifs sont mis à incuber :

- 10 flacons sont diposés sur un profil vertical (profil de photosynthèse classique) (STRICKLAND, 1960, DAUTA et CAUDERLIER, 1984). Le milieu est donc non-agité :

- 1 flacon mobile entre la surface et le fond du bassin (220 rotations. $\min ^{-1}$ ). Sa production est considérée comme la moyenne d'un profil vertical agité. 
Ces mesures ont été répétées au cours de l'année, entre septembre 1989 et mai 1990. De plus, un suivi de la production a été réalisé entre le lever et le coucher du soleil.

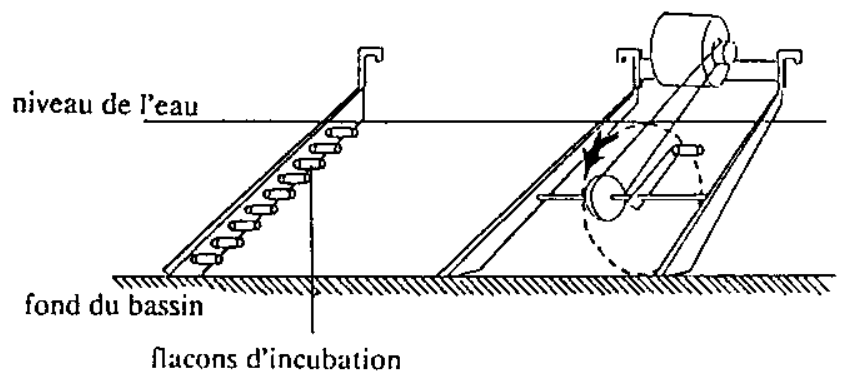

Figure 1 Dispositifs utilisés pour mesurer la production primaire : sans agitation avec agitation.

Device used for primary productivity measurements : with and without mixing.

\section{RÉSULTATS}

\section{Variations sur une période de 24 heures}

Huit expériences de mesures d'activité photosynthétique (1 à 2 heures d'incubation) ont été effectuées entre le lever et le coucher du soleil pendant une journée très ensoleillée en août 1989 (Intensité lumineuse maximale moyenne de $588 \mu \mathrm{E} \cdot \mathrm{m}^{-2} \mathrm{~s}^{-1}$ ).

Dans le bassin considéré, les paramètres physico-chimiques, homogènes sur la verticale, montrent de fortes variations sur 24 heures (fig. 2):

-15 à $22^{\circ} \mathrm{C}$ pour la température.

$-6,8$ à 8,3 pour le $\mathrm{pH}$,

-4 à $16 \mathrm{mg} \cdot .^{-1}$ pour l'oxygène dissous,

les plus hautes valeurs sont observées au milieu de l'après midi, et les plus basses à l'aube. Les activités photosynthétiques sont mesurées dans un bas$\sin$ (espèces indigènes diverses) présentant les caractéristiques suivantes :

- coefficient d'extinction (KE) $=-17 \mathrm{~m}^{-1}$

- chlorophylle (moyenne sur la journée) $=1,04 \mathrm{mg} . \mathrm{I}^{-1}$

- alcalinité = $295 \mathrm{mg} \mathrm{CaCO}_{3} \cdot 1^{-1}$ 

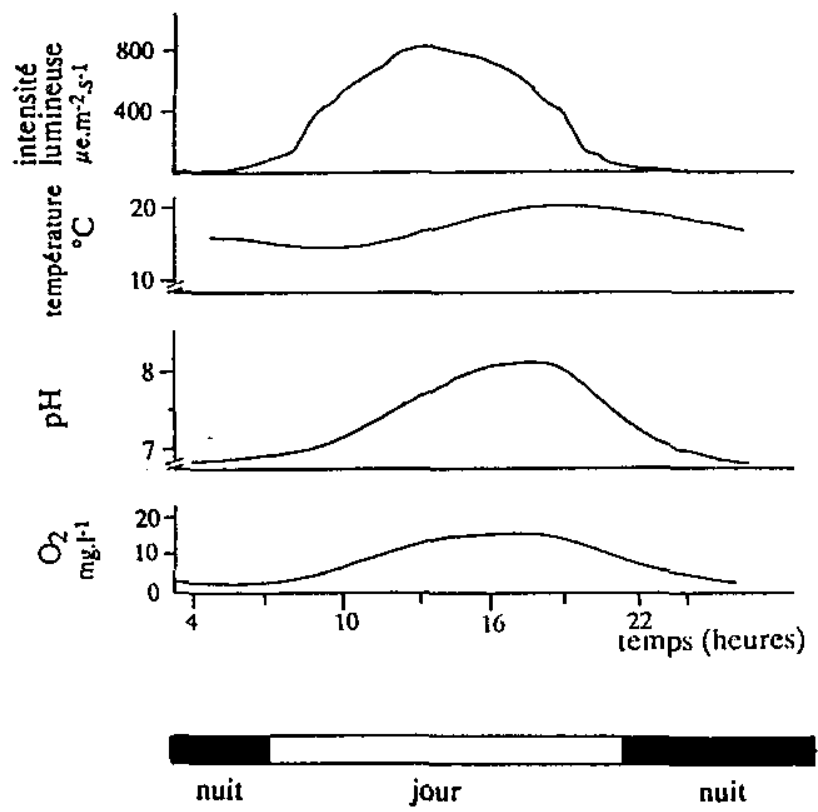

Figure 2 Paramètres physico-chimiques enregistrés sur 24 heures dans le bassin d'incubation (homogènes sur la verticale).

Physico-chemical parameters registred over 24 hours in incubation pond (homogeneous on the vertical of the pond).

La valeur élevée de KE est liée aux densités importantes de chlorophylle. qui peuvent atteindre $2 \mathrm{mg} . \mathrm{t}^{-1}$ dans les bassins de haut rendement.

La photosynthèse commence dès le lever du soleil, et est efficace jusquau coucher du soleil. Le profil de photosynthèse obtenu avec le système stationnaire (fig. 3) montre que :

- à 7 h 00 , avec une énergie lumineuse de $91 \mu \mathrm{E} \cdot \mathrm{m}^{-2}$, la photosynthése est déjà active. Ceci explique la remontée rapide de l'oxygène juste après l'aube, alors que la lumière incidente est encore très faible. De même, la photosynthèse est active au moins jusqu'à $20 \mathrm{~h}$, soit 1 heure avant le coucher du soleil. Pendant ces périodes, la partie superficielle de l'eau est la seule productive ;

- pour les fortes intensités lumineuses, il apparaît une inhibition à la surface du bassin.

Les profils verticaux s'ajustent parfaitement au modèle de STEELE (1962, eq. 1), qui tient compte de la forte inhibition de surface, contrairement à celui de TALING (1957). 


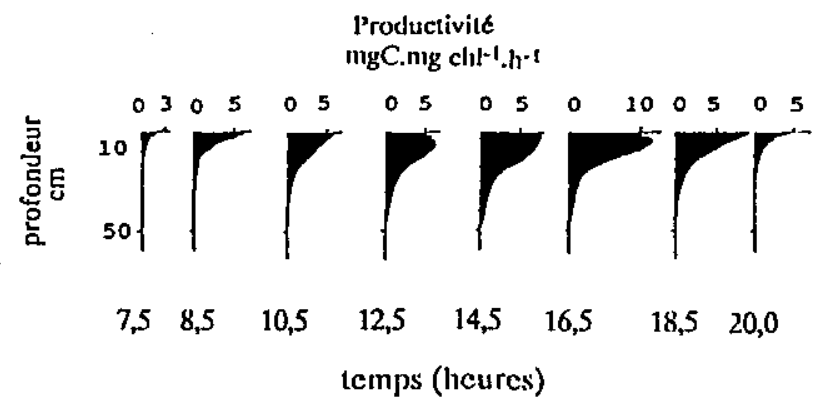

Figure 3 Production brute mesurée en fonction de la profondeur sur 24 heures (durant la période claire).

Growth production versus depth, measured over 24 hours (between sunrise and sunset).

Dans les conditions expérimentales citées précédemment (fig. 2), le taux maximum de photosynthèse est enregistré entre 16 h et 17 h $(10,6 \mathrm{mg} \mathrm{C.mg}$ $\mathrm{chl}^{-1} \cdot \mathrm{h}^{-1}$ à $5 \mathrm{~cm}$ de profondeur).

Les résultats obtenus ont été intégrés sur la profondeur et peuvent être comparés aux valeurs mesurées dans le flacon en rotation et extrapolées à la colonne d'eau (fig. 4).

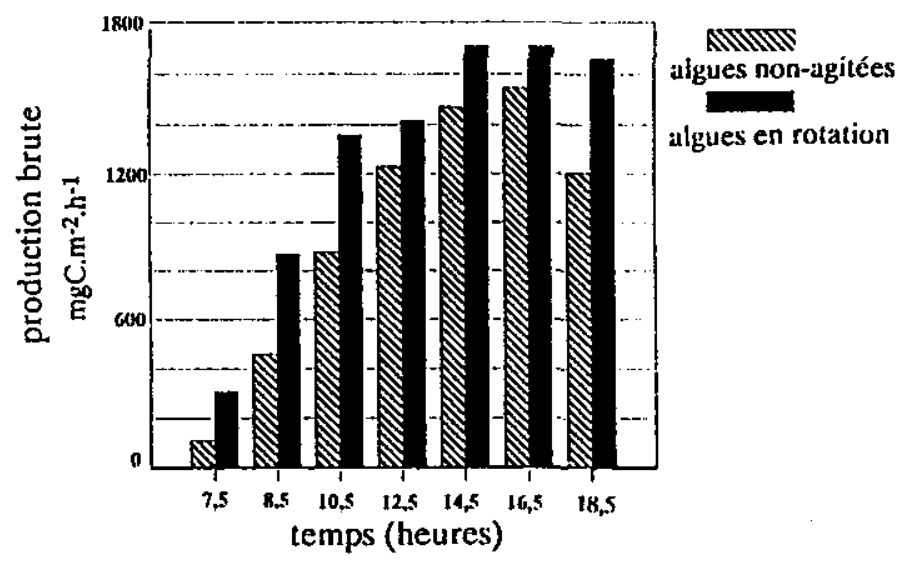

Figure 4 Comparaison de la production brute mesurée pour les deux types de profils sur 24 heures.

Growth production for the two profiles over 24 hours. 
Pour cette période de forte activité photosynthétique, les algues en mouvement ont toujours un taux instantané de photosynthèse supérieur. L'effet de l'agitation est particulièrement marqué au lever et au coucher du soleil, où la photosynthèse peut être 2,5 fois supérieure pour les algues en mouvement.

La figure 4 présente la productivité brute des algues cumulée sur une journée. La production photosynthétique brute instantanée par unité de surface de plan d'eau $\left(\mathrm{m}^{2}\right)$ est estimée en intégrant les profils verticaux de photosynthèse, à partir des valeurs de photosynthèse calculées avec l'équation (1) pour des tranches d'eau de $0,5 \mathrm{~cm}$; la production totale est ensuite calculée par intégration. L'intensité lumineuse au niveau de chaque profondeur $z$ (Ip) est définie par la relation:

$$
1 p=I_{0}^{*} \exp ^{-k z}
$$

avec:

$\mathrm{KE}=$ coefficient d'extinction de la lumière dans l'eau,

et $I_{0}=$ lumière incidente en surface.

\section{Variations sur l'année}

La production photosynthétique mesurée par les deux méthodes (fig. 5) peut être comparée :

- au cours d'une journée,

- au cours de l'année.

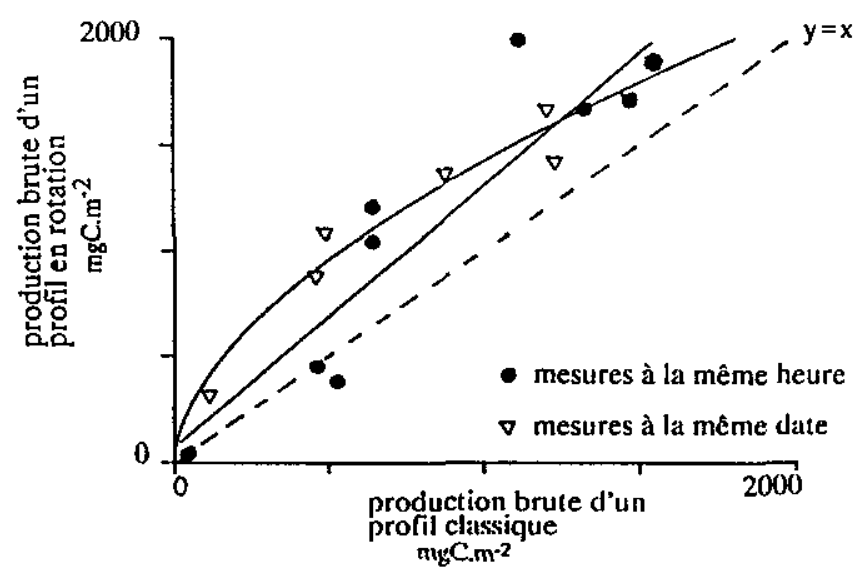

Figure 5 Représentation de la relation entre les deux profils de photosynthèse mesurés soit pour une même date (pendant 24 heures), soit pour une même heure (pendant l'année).

Relation between the two profiles for a same date (over 24 hours) or for a same hour (over the year). 
Dans le premier cas, les deux valeurs de production sont liées par la relation :

$$
\mathrm{Pbr}=27,8^{*} \mathrm{Pbc}^{0,57}
$$

$(r=0,952, n=8)$

$\mathrm{Pbr}=$ production brute du profil rotatif $\left(\mathrm{mg} \mathrm{C} . \mathrm{m}^{-2} \cdot \mathrm{h}^{-1}\right)$

$\mathrm{Pbc}=$ production brute de profil classique $\left(\mathrm{mg} \mathrm{C} \cdot \mathrm{m}^{-2} \cdot \mathrm{h}^{-1}\right)$.

Tandis que sur l'année, la relation mettant en cause divers taux d'activité photosynthétique peut être considérée comme linéaire :

$(r=0,93, n=10)$.

$$
\mathrm{Pbr}=1,24^{*} \mathrm{Pbc}+70,2
$$

Pour des périodes de faibles activités photosynthétiques (hiver), la production en milieu non-agité est supérieure.

Nous avons représenté les productions brutes observées en fonction de la profondeur, et cherché la représentation mathématique la plus proche des points observés (avec calcul par les équations 1 et 2 de Pmax et lopt). A partir de cette représentation, les productions nettes sur 24 heures ont été calculées par cumul suivant un pas de 0,5 heure, en estimant que les pertes par respiration sont sensiblement égales à $10 \%$ de Pmax quelle que soit la période du jour (HARRIS, 1984). L'intensité de surface à chaque heure $\left(I_{0 h}\right)$ est calculée d'après la formule suivante :

$$
I_{\text {oh }}: I \max * \sin \left[\pi^{*}(H-H L) / P P\right]
$$

$H=$ heure du jour,

$H L=$ heure du lever du soleil,

$P P=$ photopériode,

et Imax = Intensité maximale du jour concerné.

La figure 6 donne un exemple de la production nette des milieux agités et non-agités sur une journée, et le tableau 1 compare les productions nettes, sur une année. Fin avril, le profil agité devient plus productif que le profil nonagité.

Tableau 1 Comparaison des productions nettes obtenues pour les deux profils à différentes époques de l'année en $\mathrm{gC} \cdot \mathrm{m}^{-2} \cdot \mathrm{j}^{-1}$.

Table 1 Net production ( $\left.g C . m^{-2} . \sigma^{-1}\right)$ of the two profiles for various times over the year.

\begin{tabular}{|cccc|}
\hline Date & four & Profll classique & Profll rotation \\
\hline $6 / 03 / 90$ & 65 & 0,2 & 0,18 \\
$4 / 04 / 90$ & 94 & 2,86 & 2,04 \\
$27 / 04 / 90$ & 117 & 6,8 & 17,9 \\
$9 / 08 / 89$ & 221 & 3,67 & 17,35 \\
$22 / 08 / 89$ & 234 & 4,83 & 18,14 \\
$29 / 08 / 89$ & 241 & 4,9 & 17,86 \\
\hline
\end{tabular}




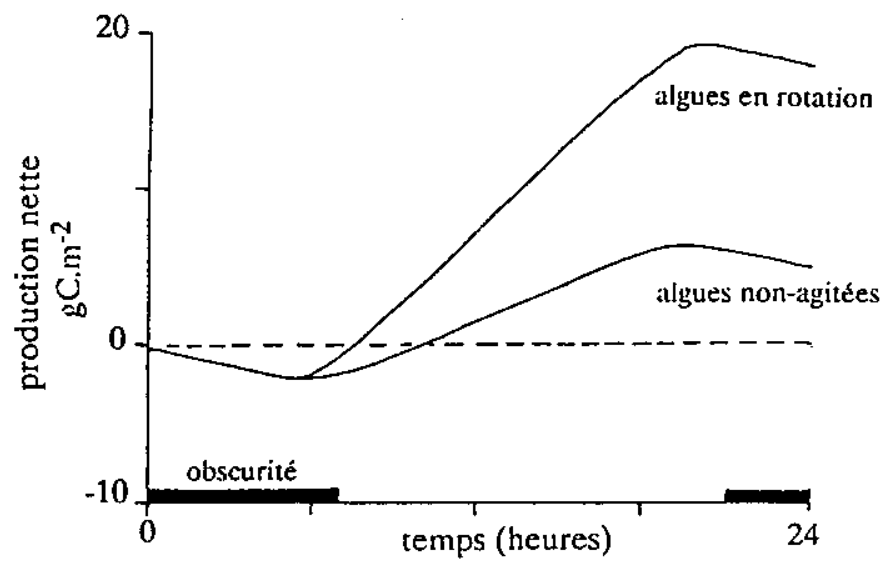

Figure 6 Production nette cumulée sur 24 heures pour les algues en agitation ou stationnaires

Net production over 24 hours for algae without or with mixing.

\section{DISCUSSION}

Ces résultats doivent être considérés en tenant compte des diverses techniques d'agitation. Les roues à aubes, plus couramment utilisées pour la culture en masse à l'extérieur, créent un flux turbulent avec une vitesse linéaire (qui respecte la zone photique), bien qu'il entraîne un changement continuel dans la position des algues (RICHMOND et BECKER, 1986). Pour opti miser la production, LAWS et al. (1983) ont construit un système de production dans lequel l'agitation s'effectue suivant des mouvements tourbillonnants, utilisant l'effet flash.

Nos bassins expérimentaux ont une profondeur de $0,5 \mathrm{~m}$ et une largeur de $1 \mathrm{~m}$, si bien que les roues à aube induisent un mouvement qui ne respecte pas les couches photiques et est proche du tourbillon. Les résultats obtenus doivent donc expliquer les phénomènes observés dans une telle culture. Cette étude permet d'estimer sur 24 heures le gain en production primaire : il est particulièrement élevé (pour les périodes de fortes activités photosynthétiques), lorsque la photosynthèse démarre ou diminue. Nous pouvons distinguer trois causes aux effets positifs de l'agitation :

- pour les fortes intensités lumineuses, la photoinhibition diminue ou disparaît (RAVEN, 88) : 
- pour les faibles intensités lumineuses, avec un point de compensation proche de la surface, la mince couche d'eau productive d'un milieu non-agité est remplacée par une colonne d'eau homogene avec une forte production ;

- quelle que soit l'intensité lumineuse, "l'effet de flash " apparaît comme une action positive additionnelle. Ces résultats sont en accord avec ceux de LITTLE (1964), dont les expériences montrent qu'une périodicité de flashs lumineux de l'ordre de la seconde augmente significativement l'efficacité photosynthétique en comparaison avec un périodicité des flashs de $10 \mathrm{~ms}$.

Aussi, pour une culture agitée très productive avec une couche photique faible, si l'on considère le cycle journalier et la profondeur du point de compensation, il se produit une variation continue pour la durée des flashs lumineux: dans la journée, la durée des flashs diminue avec l'accroissement de l'intensité lumineuse (la lumière pénètre sur une plus grande profondeur, et les flashs sont donc plus longs pour une algue donnée. Par contre, puisque la vitesse de rotation est constante, la périodicité ne varie pas).

D'autres facteurs agissent également sur l'activité photosynthétique $\left(\mathrm{PO}_{2} / \mathrm{PCO}_{2}\right.$ par exemple, MARKL, 1977), mais dans les conditions expérimentales, la lumière est sans doute le principal facteur influent : pour le profil en agitation, les conditions d'oxygène, de $\mathrm{pH}$ et de température sont proches de celles du profil non-agité (la production est du même ordre de grandeur dans les deux cas, et les algues sont confinées sur un court laps de temps dans des flacons de $20 \mathrm{ml}$ ). Par contre, il est vrai que la méthode expérimentale ne tient pas compte des effets physiques de l'agitation sur les cellules élles-mêmes, effets dont on ne peut connaître limpact sur la photosynthèse.

Pour les périodes de faibles activités synthétiques (hiver), l'effet de flash ne semble pas jouer ; ceci explique que la production en mitieu agité soit plus faible. En mars 1990 par exemple, la profondeur de compensation mesurée était de $90 \mathrm{~cm}$ : quel que soit le mouvement des algues, la lumière était disponible jusqu'au fond du bassin. Une explication au fait que la production du flacon en rotation soit plus faible, peut être la suivante : puisqu'il n'y a plus de zone d'ombre, l'effet de flash n'est plus efficace.

Les résultats présentés ici donnent une estimation des gains enregistrés par l'agitation de cultures d'algues denses et pourront donc être pris en considération pour le monitoring des cultures algales en milieu extérieur, lorsque l'on souhaite optimiser la production

\section{RÉFÉRENCES BIBLIOGRAPHIQUES}

DAUTA A., CAUDERLIER E., 1984. Mesure de production primaire in situ : un systeme d'incubation adapté aux milieux à tortes concentrations algales. In Association Fran- çaise pour rAlgologie Appliquée Ed., Actes du colloque de L'AFAA, 225-234.

HARRIS G.P., 1984. Phytoplanikton productivity and growth measurements : Past, 
present and future. J. Plankton Res., 6. 219-237.

KOK B., 1953. Experiments on photosynthesis by Chlorella in flashing light. In J.S. BURLEW Ed., Algal Cultures : From Laboratory to Pilot Plant, publ. $n^{\circ} 600$. The Carnegie Institution Wasthington.

LAWS E., TERRY K., WIEKMANN J., CHALUP M., 1983. A sample algal production system designed to utilize the flashing light effect. Biotechnol Bioeng., 25, 2319-2335.

LITTLE A., 1964. Pilot plant studies in the production of Chlorella. In J.S. BURLEW Ed., Algal cultures : From Laboratory to Pilot Plant, publ. $n^{\circ} 600$. The Camegie Institution, Washington, 235.

MARKL H., 1977. CO2 transport and photosynthetic productivity of a continuous culture of algae. Biotechnol. Bioeng., 19, 1851-1862.

MARKL. H., 1980. Modelling of algat produciton systems. In G. SHELEF and C.J. SOEDER Eds., Algae Biomass, Elsevier/North Holland Biomedical Press, Amsterdam, 361-383.

MARKER A., NUSH E., RIEMANN B., 1980. The measurements of photosynthetic pigments in freshwater and standardization of methods : conclusions and considerations. Erg. Limnol., 14, 91-106.

MYERS J., GRAHAM J.R., 1971. The photosynthetic unit in Chlorella measured by repetitive short flashes. Plant physiology, 48, 282-286.

MYERS J., 1980. On the algae : thoughts about physiology and measurements of efficency. In P.G. FALKOWSKI Ed., Primary productivity in the sea, Plenum Press, New York, 1-16.
OSWALD W.J., 1988. Large scale algal culture systems (engineering aspects). In M.A. BOROWITZKA and L.J. BOROWITZKA Eds., Micro-algal biotechnology, Cambridge University Press, Cambridge, 357-394.

RAVEN J.A., 1988. Limits of growth. In M.A. BOROWITZKA and L.J. BOROWITZKA Eds., Micro-algal biotechnology, Cambridge University Press, Cambridge, 331. 356.

RICHMOND A., VONSHAK A., 1978. Spirulina culture in Israël. Arch. Hydrobiol. Beih. Erg. Limnol., 11, 274.

RICHMOND A., BECKER E., 1986. Technological aspect of mass cultivation. A general outline. In A. RICHMOND Ed., Hanbook of micro-algal mass culture, CRC Press, Boca Raton, Florida, 245-263.

STEELE J.H., 1962. Environmental control of photosynthesis in the sea. Limnol. Oceanogr., 7, 137-150.

STRICKLAND J.D., 1960. Measuring the production of marine phytoplankton. Bull. Fish. Res. Bd. Can., 15, 453-493.

TALLING J.F., 1957. The phytoplancton population as a compound of photosynthetic system. New. Phytol., 56, 133-149.

TAMIYA H., SHIBATA K., SASA T., IWAMURA T., MORIMURA Y., 1953. Effect of diurnally intermitent lumination on the growth and some extracellutar characteristics of Chlorella.. In J.S. BURLEW Ed., Algal cultures : From laboratory to pilot plant, publ. $n^{\circ} 600$, The Carnegie Institution, Washington.

WINOKUR M., 1948. Gowth relationship of Chlorella species. American J. Bot., 35. 118-129. 